NUCLEAR INSTRUMENTS AND METHODS 62 (I968) 295-297; (C) NORTH-HOLLAND PUBLISHING CO.

\title{
MAGNETOSTRICTIVE READOUT OF SPARK CHAMBERS IN A MAGNETIC FIELD*
}

\author{
M. L. MARSHAK and S. M. PRUSS
}

Randall Laboratory of Physics, University of Michigan, Ann Arbor, Michigan, U.S.A.

Received 1 February 1968

\begin{abstract}
Magnetic fields of moderate strength may cause errors of order $0.5 \%$ in measurements made with magnetostrictive spark chamber
\end{abstract} readouts.

The fringe magnetic field surrounding momentumanalyzing magnets will alter the velocity, amplitude and shape of the output pulses of magnetostrictive spark chamber readouts located near these magnets. These effects are always adverse to the proper digitizing of spark chamber coordinates. The behavior of the pulses depends on the direction and strength of the ambient magnetic field at the send and intermediate areas of the magnetostrictive delay line and on the intrinsic characteristics of the line.

The effect of ambient magnetic fields on the received amplitude of longitudinal-mode pulses has previously been reported for several ferromagnetic materials ${ }^{1}$ ). The current measurements, also for longitudinal-mode pulses, extend this data for a commonly-used magnetostrictive alloy to include external stress as an additional parameter and pulse velocity and shape as observables. Since this magnetostrictive material has properties which vary considerably from batch to batch, emphasis is placed on the qualitative effects which occur and the order of magnitude of these effects.

The samples of "Remendur", ) which we evaluated were derived from two independent batches manufactured to similar specifications, except that one batch was annealed at $550^{\circ} \mathrm{C}$ while the other was not annealed. The delay lines all measured $0.004^{\prime \prime} \times 0.015^{\prime \prime}$. The magnetostrictive pulses were produced by a single turn of wire wrapped around the "Remendur" line and driven by a spark gap at approximately $5 \mathrm{kV}$; the receive coil was 200 turns of no. 50 wire wound on a plastic die approximately $1 \mathrm{~mm}$ wide and $2 \mathrm{~mm}$ in diameter, mounted concentrically on the delay line. The output of the coil was displayed directly on an oscilloscope. The external stress on the "Remendur" ribbon was applied through a hanging weight arrangement.

The velocity of the longitudinal magnetostrictive strain pulse in our samples of "Remendur" was 5.4 $\pm 0.1 \mathrm{~mm} / \mu \mathrm{sec}$ at room temperature with no external magnetic field or stress on the ribbon. The velocity is equal to $(E / \rho)^{\frac{1}{2}}$ where $E$ is Young's modulus and $\rho$

* Supported by the U.S. Atomic Energy Commission. is the linear mass density ${ }^{3}$ ). For practical purposes the velocity can be considered frequency independent; no dispersion was noticed over the length of the half meter delay line used for these measurements.

The variation in pulse velocity $(\Delta v / v)$ with an external magnetic field or an external stress was of order $\pm 0.5 \%$ for our samples. An increase in velocity with an increase in field was observed in the "Remendur" that had been annealed; a decrease in velocity with an increase in field was observed for the "Remendur" that had not been annealed. The magnitude of the dependence was similar for all samples; the velocity change was approximately 10 times more dependent on the magnitude of the longitudinal field than on the magnitude of the transverse field and independent of the sign of either field. For both longitudinal and transverse fields the velocity dependence on field was approximately linear - at least for the fields of up to several kilogauss which we were able to produce. The velocity change for our samples did not depend on the previous stress history of the delay line. Figs. 1 and 2 indicate the dependence of the delay characteristics of the magnetostrictive line on the external field. Measurements were made by varying both the applied field and the length of delay line. The results by both methods agree with the plotted curves.

The effect of tension on velocity for both samples was of the same sign as the effect of an external field. We found that for any tension that might actually be encountered in a magnetostrictive spark chamber readout the velocity dependence on tension for delay lines up to several meters long was less than the error in discrimination on the signal peak (about $100 \mathrm{nsec}$ ). By using large external tensions (more than 500 newton/ $\mathrm{mm}^{2}$ ), the velocity change can be made to exceed $0.5 \%$.

The shape of the pulse output by the receive coil on a magnetostrictive delay line with neither external field nor tension is proportional to the second time derivative of the spark send pulse ${ }^{3}$ ). The observed shape shown in fig. 3a approximates the second derivative of a gaussian. 


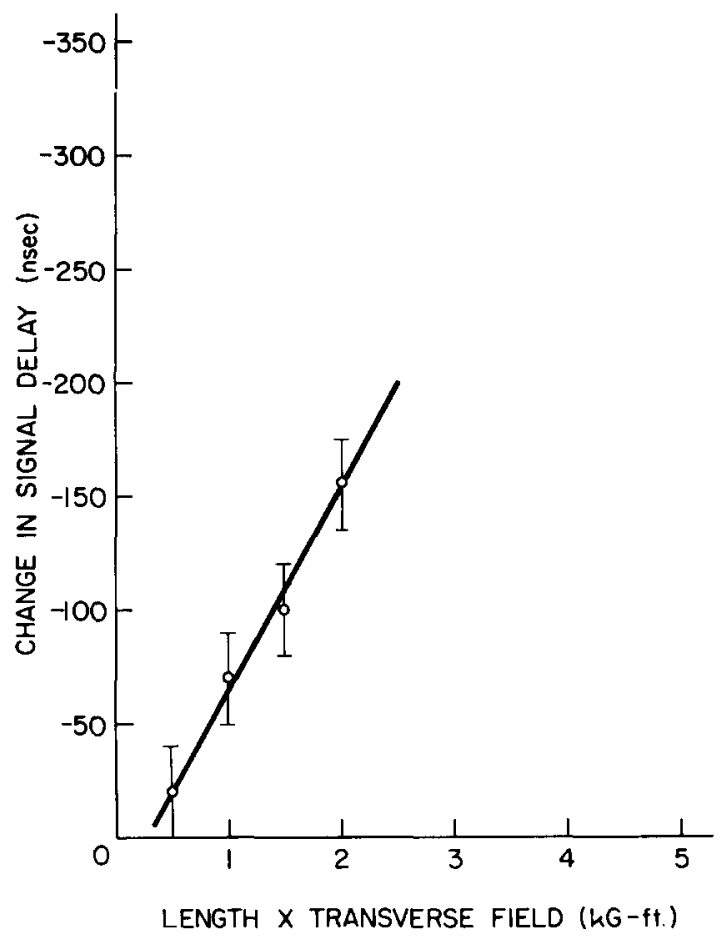

Fig. 1. The variation in signal delay $(A D)$ as a function of the transverse magnetic field for the annealed "Remendur".

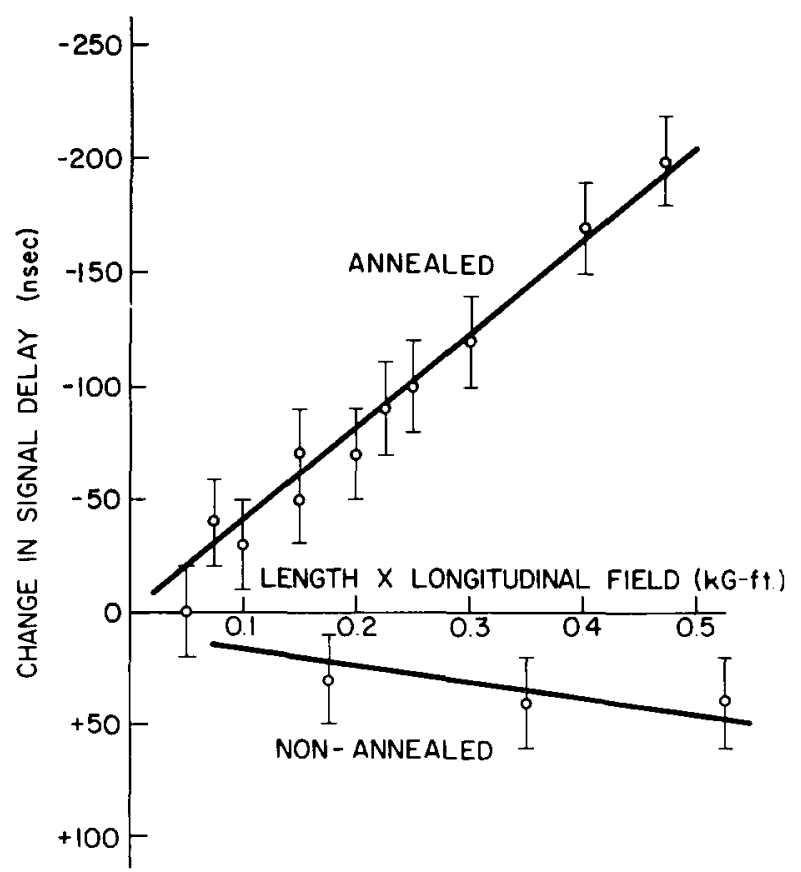

Fig. 2. Variation in signal delay $(\Delta D)$ as a function of the longitudinal magnetic field for both annealed and unannealed "Remendur".
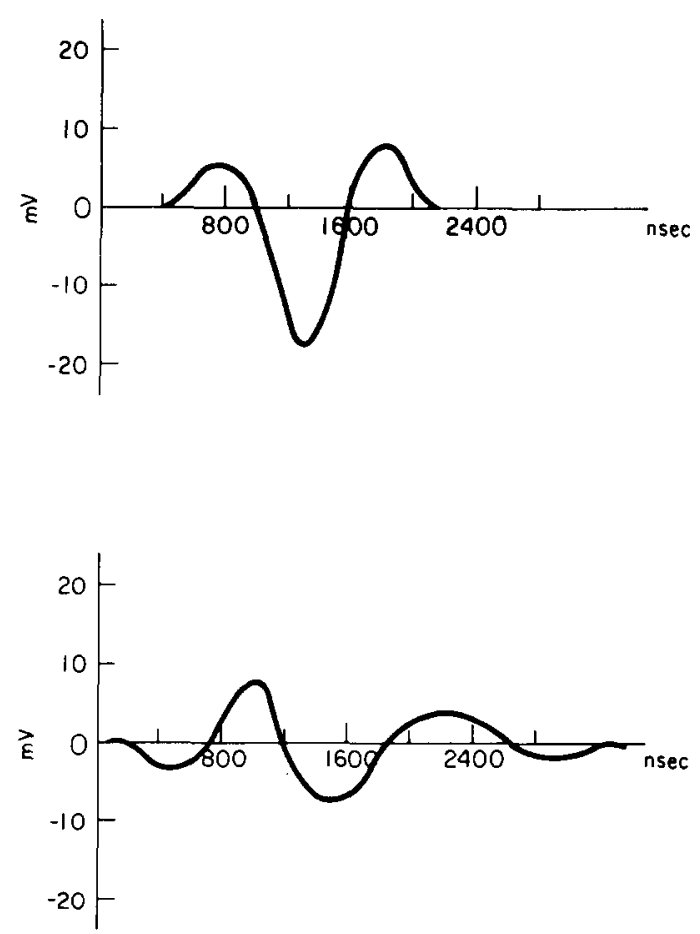

Fig. 3. a. Typical receive coil ouput pulse shape with neither external magnetic field nor tension on the delay line; b. Typical receive coil output pulse shape distorted by an external magnetic field or tension.

The polarity of the output pulse is determined by the direction of the send current and the direction of the longitudinal components of the external magnetic fields at both the send or receive areas; changing any one of these parameters inverts the pulse.

The amplitude of the received pulse is damped by the application of an external field or stress to the magnetostrictive delay line. Both Kaufman ${ }^{1}$ ) and the present authors find that the amplitude of the central maximum of the received pulse is relatively insensitive to small transverse fields; the signal is not measurably diminished for transverse fields of less than $3 \mathrm{kG}$ at either the send or intermediate areas of the delay line. The longitudinal-mode pulse amplitude for both the annealed and unannealed lines depends much more critically on the maximum external longitudinal field; the amplitude is also quite insensitive to either the field gradient or the length of delay line in the longitudinal field. The pulse amplitude for both the annealed and the unannealed delay lines decreases to $50 \%$ of the no-field amplitude for a longitudinal field to approximately $200 \mathrm{G}$. Upon inverting the direction of the longitudinal field the amplitude follows a hysteresis curve about $60 \mathrm{G}$ wide because of the magnetization of the delay line itself. 


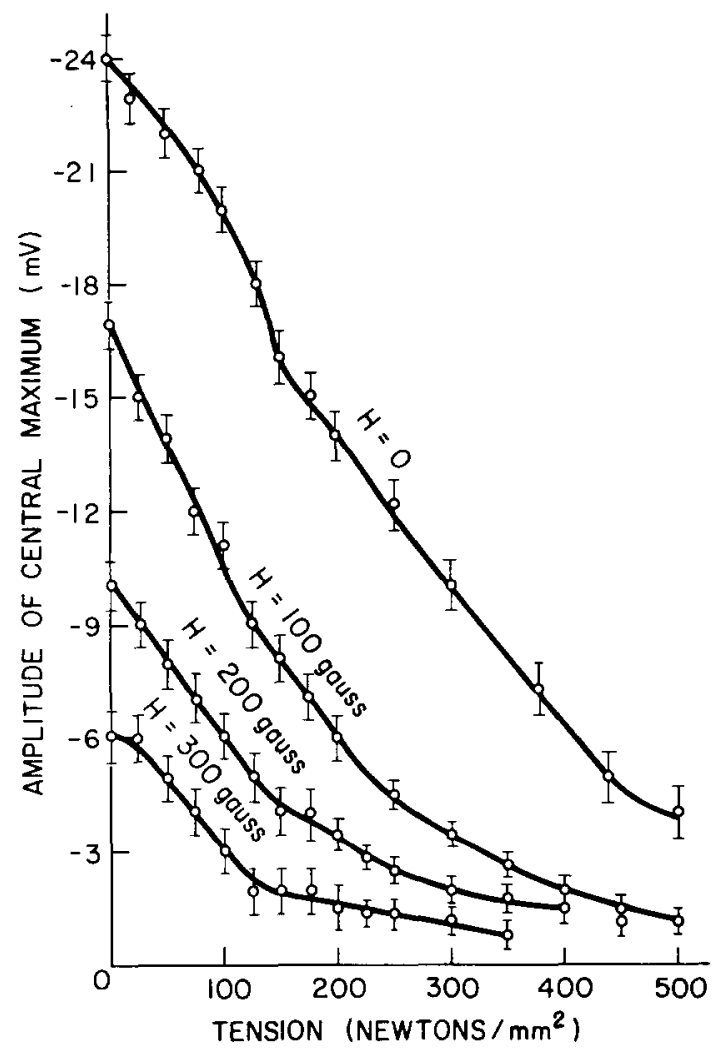

Fig. 4. Amplitude of central maximum of receive coil output pulse for unannealed "Remendur" as a function of tension and longitudinal magnetic field in a $20^{\prime \prime}$ solenoid.
The damping of the strain wave packet by the external field is frequency dependent, as would be expected from arguments considering the magnetic coupling of the delay line to the inductance of the field-producing magnet. The qualitative change in the wave packet shape is shown in fig. 3b. As a general rule, if the central maximum has decreased by more than $50 \%$ of its zero-field amplitude the receive coil output pulse shape is so altered that it is no longer suitable for timing discrimination.

An external tension on the wire damps the amplitude of the output pulse. The magnetic and tension damping are both shown in fig. 4 . In a manner similar to the magnetic field effect, the damping by external tension is dependent on the frequency of the various Fourier components of the strain wave packet.

The authors wish to thank Professor D. I. Meyer for many helpful discussions and for reading the manuscript.

\section{References}

1) L. Kaufman, V. Perez-Mendez and J. Pfab, IEEE Trans. Nucl. Sci. 13 (1966) 578.

2) "Remendur" is an alloy of $49 \%$ iron, $49 \%$ cobalt and $2 \%$ vanadium manufactured by Wilbur B. Driver Company, Newark, New Jersey.

3) V. Perez-Mendez and J. Pfab, Nucl. Instr. and Meth. 33 (1965) 141. 